\title{
On the interaction of a submerged turbulent jet with a clean or contaminated free surface
}

\author{
Douglas G. Anthony \\ David Taylor Research Center, Bethesda, Maryland 20084 \\ Amir Hirsa and William W. Willmarth \\ Department of Aerospace Engineering, The University of Michigan, Ann Arbor, Michigan 48109
}

(Received 3 August 1990; accepted 22 October 1990)

\begin{abstract}
The effect of a free surface on the structure of a submerged turbulent jet is investigated experimentally. Three-component LDV measurements beneath a clean free surface show that the mean flow spreads laterally outward in a shallow surface layer much wider than the mean flow well below the surface. As the free surface is approached, velocity fluctuations normal to the surface are diminished while those parallel to the surface are enhanced.

Laser-induced fluorescence is used to show that the surface layer contains fluid ejected from the jet. With the addition of surface-active agents, the surface layer is suppressed.
\end{abstract}

The interaction of vortical flows, especially turbulent flows, with a free surface is of considerable interest to the detection and determination of the hydrodynamic signature of surface ships. Recent research investigating the interaction of laminar axisymmetric vortex rings with the free surface has revealed that vortex disconnection at the surface and the subsequent alignment of vorticity normal to the surface are a fundamental physical mechanism governing the interaction. ${ }^{1}$ Investigations of the interaction of a laminar two-dimensional vortex pair with clean and contaminated free surfaces ${ }^{2,3}$ have shown vortex rebounding associated with the generation of secondary vorticity beneath the contaminated surface; the generation of secondary vorticity and the rebounding of the primary vortex are not observed when the surface is clean. The degree to which these physical phenomena, observed in laminar flows, govern the interaction of turbulence with a free surface is the subject of much current research.

Observations of a submerged turbulent jet issuing beneath a free surface have bcen reported by Bernal and Madnia. ${ }^{4}$ Shadowgraphs of the free surface showing deformations that occur during the interaction of the jet with the surface reveal surface dimples, formed where vortex lines become connected to the free surface, and waves, observed to propagate outward and downstream of the point of their generation. Quantitative measurements ${ }^{4}$ of the streamwise velocity component were made using hot-film anemometry, but accurate measurements close to the free surface were difficult because the sensor was intermittently in and out of the water. Also, the effects of surface contamination were not taken into account.

In the present investigation, simultaneous threecomponent velocity measurements in an initially circular, turbulent jet discharging beneath and parallel to a clean free surface were made using a laser Doppler velocimeter (LDV). The nonintrusive nature of this technique made measurements of the flow velocity just beneath the surface possible. These measurments reveal that the turbulent velocity fluctuations normal to the free surface are diminished just beneath the surface, while those parallel to the free surface are enhanced, as shown in Fig. 1. Similar behavior has been reported for channel flow ${ }^{5,6}$ and for twodimensional surface-jet flow. ${ }^{7}$

Measurements of the mean flow in a cross-sectional plane at $x / d=32$ for a jet at depth $h / d=2$ beneath a clean free surface are displayed in Fig. 2. Shown are the horizontal and vertical components of velocity both within the turbulent jet flow and extending laterally beneath the surface to about two jet half-widths away. A thin surface current layer is clearly visible, as is the abrupt transition from inward (entraining) flow beneath the layer to outward flow within it. We suggest that the surface current is caused by the ejection of vortical structures from the jet and the subsequent outward propagation, near the surface, of a portion of these structures under the influence of their corresponding images above the surface. Superposed on this layer are surface waves that propagate away from the jet centerline. The Reynolds number based on jet exit velocity and diameter was $U_{e} d / v \approx 13000$, and the Froude number based on jet exit velocity and depth was $U_{e}(g h)^{1 / 2} \approx 5.7$.

The mean flow pattern of Fig. 2 is suggestive of a streamwise vortex pair with each vortex lying just outboard of the jet on either side. The observed flow pattern may arise in a region of anisotropic turbulent flow near a free surface where mixing normal to the surface is greatly reduced, allowing turbulent fluid beneath the surface to propagate a considerable distance in a thin surface layer. Similar patterns in the mean flow appear in the wakes of surface ships and are sometimes attributed to large streamwise vortices shed from the bilges. ${ }^{8}$

Figure 3 shows a cross-sectional plane at $x / d=32$ of dyed fluid emitted from the jet. The fluid is made visible using fluorescein dye which is illuminated with a laser light sheet. The free surface was cleaned by drawing off through a standpipe as much of the naturally occurring surfactants as possible. The photographs show dyed fluid ejected from the jet just beneath the free surface that has propagated away from the jet centerline, forming a thin surface layer much wider than the turbulent jet flow beneath it. The 


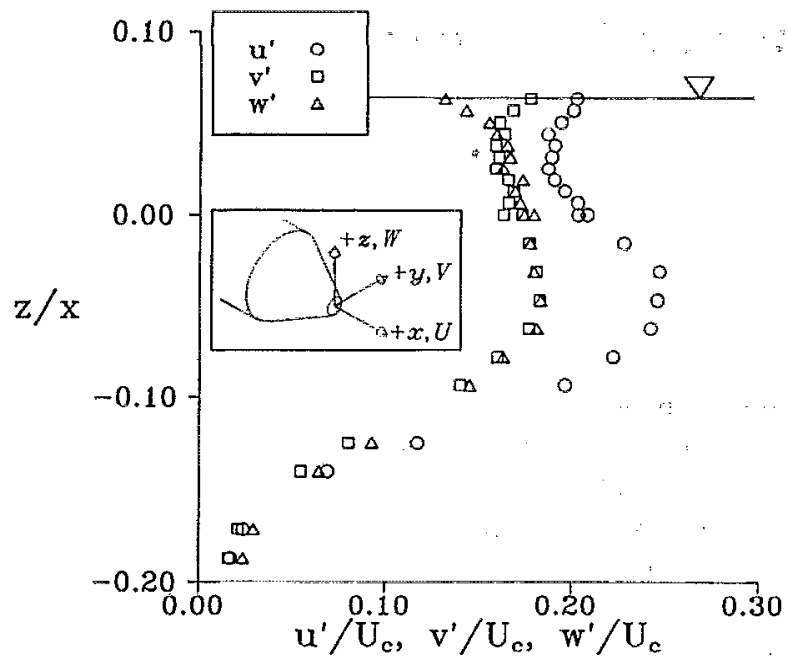

FIG. 1. Profiles of the rms velocity fluctuations beneath a clean free surface, $x / d=32, y / d=0$. The solid line indicates the position of the free surface. The coordinate system is shown in the inset, $x$ positive downstream, $z$ positive toward the free surface.

ejected fluid appears to have propagated laterally under the influence of its corresponding image fluid above the free surface, yielding a mean surface current.

Figure 4 shows the same cross-sectional plane beneath a free surface contaminated with oleyl alcohol, an insoluble surfactant for which the state relationship between surface tension and concentration is known. The concentration of surfactant was $1.3 \times 10^{-7} \mathrm{~cm}^{3} / \mathrm{cm}^{2}$ of free surface, and the corresponding surface pressure was $9 \mathrm{dyn} / \mathrm{cm}$. When surfactant is present, the surface current layer is not observed, and fluid ejected from the jet near the surface is laterally confined. We attribute this surface confinement to the interaction of the primary vortical structures ejected from the jet with secondary vorticity of opposite sign generated beneath the contaminated surface, as discussed by Hirsa ${ }^{3}$ for the laminar vortex pair.

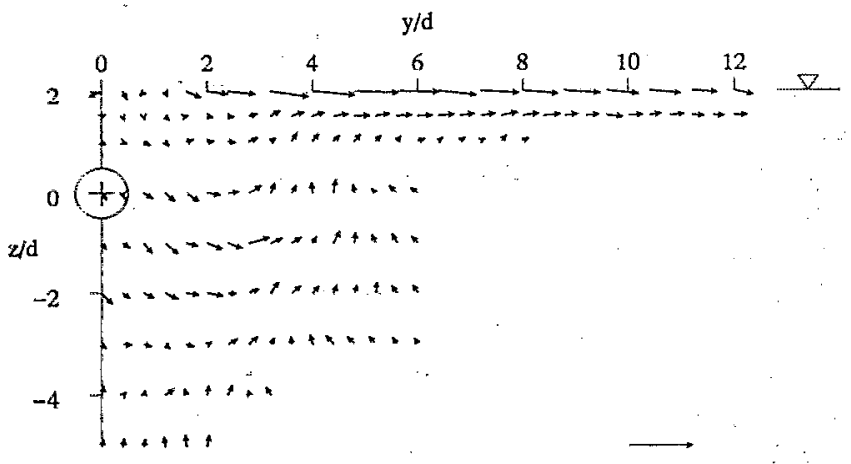

$10 \mathrm{~cm} / \mathrm{s}$

FIG. 2. Vector plot of the horizontal and vertical mean velocity components $V$ and $W$ in a cross-sectional plane at $x / d=32$ beneath a clean free surface. The shaded circle shows the $0.635 \mathrm{~cm}$ diameter jet at a depth $h / d=2$ beneath the free surface; the shaded triangle shows the location of the free surface.
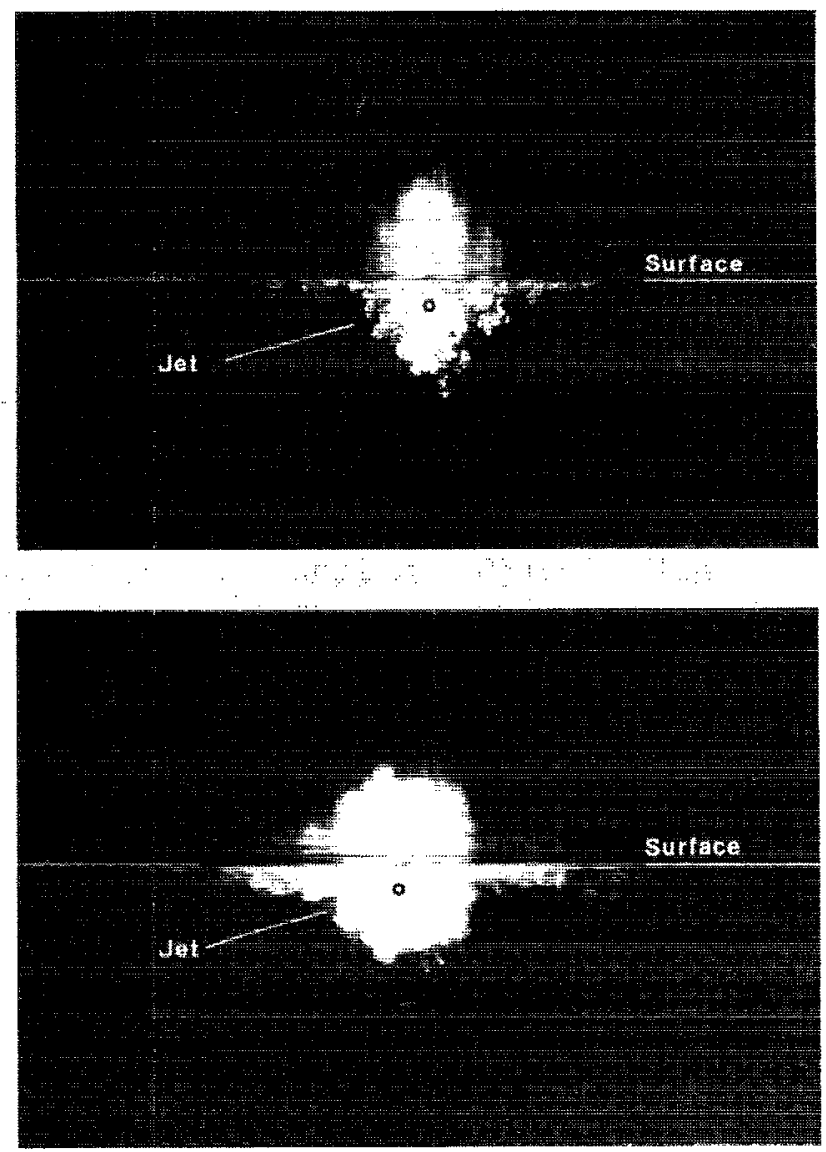

FIG. 3. Laser-induced fuorescence (LIF) photographs in a crosssectional plane at $x / d=32$ of a jet discharging beneath a clean free surface. Exposure times are $1 / 125 \mathrm{sec}$ (top) and $1 / 2 \mathrm{sec}$ (bottom).

Beneath a contaminated free surface, secondary vorticity is generated in response to gradients in surface tension, and motions of the surface continually redistribute the surfactant. In the present investigation, there was no reliable way to measure the spatial distribution of surface tension, nor to maintain a steady-state spatial distribution of surfactant concentration for sufficient time (of the order of two hours) to make velocity measurements throughout the now field. At the present state of the art, there is no way to carry out a controlled experiment; for this reason, LDV measurements of the flow velocity beneath a contaminated surface were not made.

We suggest that, near the free surface, the physical mechanism acting to transfer energy from the normal fluctuations to the fluctuations parallel to the surface is the disconnection and subsequent alignment of vortex filaments normal to the surface. The origin of the current layer beneath a clean free surface lies in the inability of this anisotropic turbulent flow to mix effectively in the direction normal to the surface. The surface current does not occur when surface active agents are present owing to the generation, just beneath the surfactant covered surface, of secondary vorticity parallel to the surface and of opposite sign to the primary (ejected) vorticity.

The present observations suggest that the physical processes observed in the interaction of discrete vortices with 

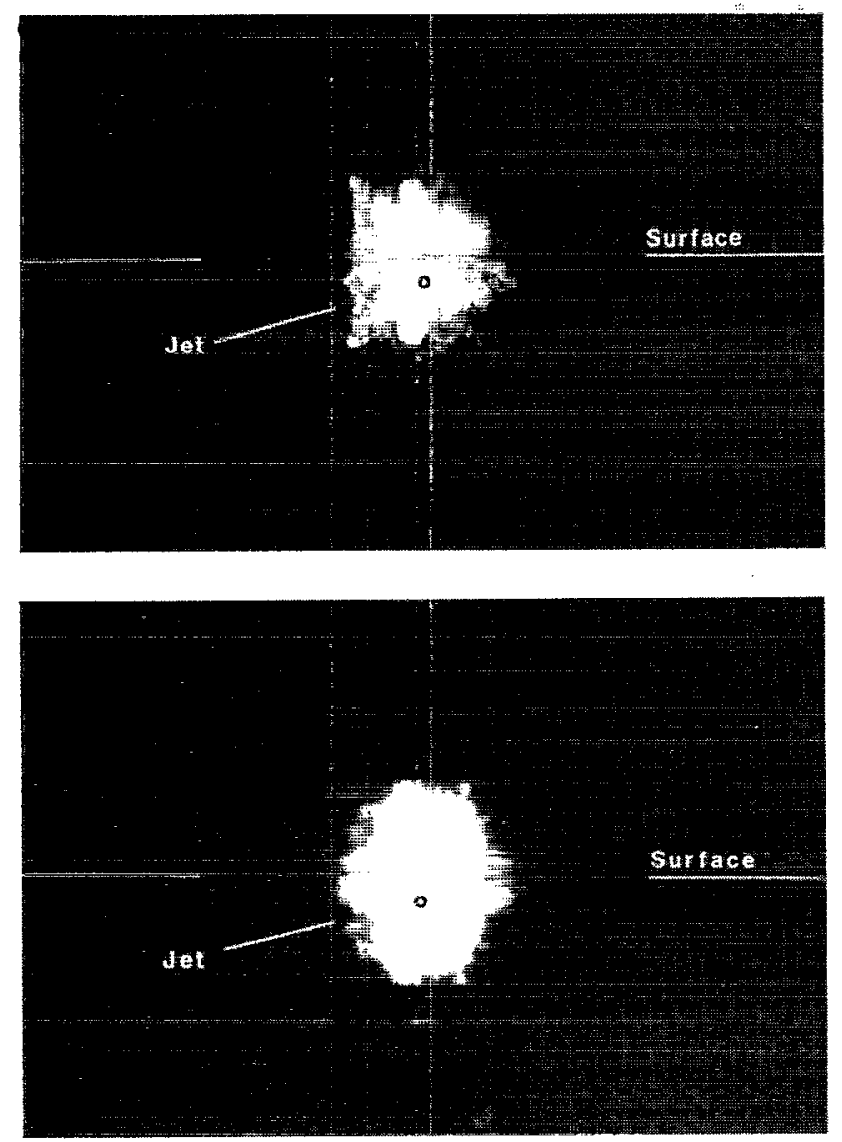

FIG. 4. LIF photographs in a cross-sectional plane at $x / d=32$ of a jet discharging beneath a free surface contaminated with oleyl alcohol. Exposure times are $1 / 125 \mathrm{sec}$ (top) and $1 / 2 \mathrm{sec}$ (bottom). the free surface, i.e., both the mechanism of vortex disconnection and alignment normal to the surface and the interaction between primary and secondary vorticity generated beneath contaminated surfaces, play fundamental roles in the interaction of fully turbulent flows with a free surface. A more complete reporting of these phenomena for a turbulent jet at depths of 2 and 50 diameters beneath a free surface, including LDV measurements of all Reynolds stress components, is in preparation.

\section{ACKNOWLEDGMENTS}

This work is supported at The University of Michigan by the Office of Naval Research University Research Initiative Program in Ship Hydrodynamics, Contract No. N000184-86-K-0684, and at the David Taylor Research Center by the ONR Surface Ship Wake Consortium Contract No. N0001490-WX-22034.

a'Present address: Department of Mechanical Engineering, Aeronautical Engineering, and Mechanics, Rensselaer Polytechnic Institute, Troy, New York 12180

'J. T. Kwon, The University of Michigan Program in Ship Hydrodynamics Technical Report No. 89-06, Ann Arbor, Michigan 1989.

${ }^{2}$ L. P. Bernal, A. Hirsa, J. T. Kwon, and W. W. Willmarth, Phys. Fluids A. 1, 2001 (1989).

${ }^{3}$ A. Hirsa, Ph.D. thesis, The University of Michigan, Ann Arbor, Michigan 1990.

${ }^{4}$ L. P. Bernal and K. Madnia, in Proceedings of the 17th Symposium on Naval Hydrodynamics, The Hague, Netherlands, 1988.

${ }^{5}$ S. Komori, H. Ueda, F. Ogino, and T. Mizushina, Int. J. Heat Mass Transfer 25, 513 (1982).

${ }^{6} \mathrm{M}$. Rashidi and S. Banerjee, Phys. Fluids 31, 2491 (1988).

${ }^{7}$ S. E. Ramberg, T. F. Swean, and M. W. Plesnia, NRL Memorandum Report 6367, Naval Research Laboratory, Washington, DC 1989.

${ }^{8} \mathrm{H}$. Lugt, Proceedings of the $3 \mathrm{rd}$ International Conference on Numerical Ship Hydrodynamics (Bassin d'Essais des Carenes Paris, France, 1981), p. 297 